Chapman University

Chapman University Digital Commons

Engineering Faculty Articles and Research

Fowler School of Engineering

6-18-2013

\title{
Spatiotemporal Fluorescent Detection Measurements Using Embedded Waveguide Sensors
}

\author{
Mark C. Harrison \\ Chapman University, mharrison@chapman.edu
}

Andrea M. Armani

University of Southern California

Follow this and additional works at: https://digitalcommons.chapman.edu/engineering_articles

Part of the Atomic, Molecular and Optical Physics Commons, Biomaterials Commons, and the Other Electrical and Computer Engineering Commons

\section{Recommended Citation}

M. C. Harrison, A. M. Armani, Spatiotemporal Fluorescent Detection Measurements Using Embedded Waveguide Sensors," IEEE Journal of Selected Topics in Quantum Electronics, vol. 20, 7000207, MarchApril 2014. https://doi.org/10.1109/JSTQE.2013.2265217

This Article is brought to you for free and open access by the Fowler School of Engineering at Chapman University Digital Commons. It has been accepted for inclusion in Engineering Faculty Articles and Research by an authorized administrator of Chapman University Digital Commons. For more information, please contact laughtin@chapman.edu. 


\section{Spatiotemporal Fluorescent Detection Measurements Using Embedded Waveguide Sensors}

\section{Comments}

This is a pre-copy-editing, author-produced PDF of an article accepted for publication in IEEE Journal of Selected Topics in Quantum Electronics, volume 20, issue 2, in 2013 following peer review. The definitive publisher-authenticated version is available online at https://doi.org/10.1109/JSTQE.2013.2265217.

\section{Copyright}

(c) 2013 IEEE. Personal use of this material is permitted. Permission from IEEE must be obtained for all other uses, in any current or future media, including reprinting/republishing this material for advertising or promotional purposes, creating new collective works, for resale or redistribution to servers or lists, or reuse of any copyrighted component of this work in other works. 


\title{
Spatiotemporal Fluorescent Detection Measurements Using Embedded Waveguide Sensors
}

\author{
Mark C. Harrison, Student Member, IEEE, and Andrea M. Armani, Senior Member, IEEE
}

\begin{abstract}
Integrated waveguide biosensors, when combined with fluorescent labeling, have significantly impacted the field of biodetection. While there are numerous types of waveguide sensors, the fundamental excitation method is fairly consistent: the evanescent field of the waveguide excites a fluorophore whose emission is detected, either directly via imaging or indirectly via a decrease in power transfer. Recently, a sensor device was demonstrated which is able to back-couple the emitted light into the waveguide, allowing the signal to be detected directly. However, this previous work focused on the development of an empirical model, leaving many theoretical questions unanswered. Additionally, the results from the novel back-coupling route were not compared with the results from the more conventional imaging technique. In the present work, we develop finite difference time domain simulations to predict the sensor's performance both in air and aqueous environments. We also perform complementary experiments to verify the modeling, measuring the fluorescence coupled into the waveguide and radiated perpendicular to the waveguide. Finally, we performed spatiotemporal measurements of the fluorescence on the waveguide. Utilizing these measurements, we are able to measure the fluorescent decay rate of the fluorescent dye at arbitrary points along the length of the waveguide.
\end{abstract}

Index Terms - modeling, waveguides, transducers

\section{INTRODUCTION}

$I^{2}$ NTEGRATED optical devices based on waveguides, resonators and lasers have been a focus of research over the past few decades and have emerged as a robust approach to performing chemical and biological sensing [1-4]. One advantage of optical sensors over other commonly used modalities, such as electrical and mechanical, is the higher resistance to environmental, electrical and mechanical noise sources, improving the signal-to-noise ratio of the device [5-

The authors would like to acknowledge Banyan Biomarkers and Dr. Brian Catanzaro for providing us with samples for testing and for helpful discussions. M. C. Harrison was supported by the Department of Defense (DoD) through the National Defense Science \& Engineering Graduate Fellowship (NDSEG) Program. The photo of M. C. Harrison was provided by Steve Cohn photography.

M. C. Harrison is with the Ming Hsieh Department of Electrical Engineering, University of Southern California, Los Angeles, CA 90089(email: mark.harrison@usc.edu).

A. M. Armani is with the Mork Family Department of Chemical Engineering and Material Science and the Ming Hsieh Department of Electrical Engineering, University of Southern California, Los Angeles, CA 90089 (e-mail: armani@usc.edu).
6]. For example, optical sensors are able to perform detection across a wide range of $\mathrm{pH}$ environments, whereas electrical sensors can have difficulties, as the $\mathrm{pH}$ can interfere with the electrical signal [7-8].

In recent years, many groups have taken upon the challenge of miniaturizing these sensing platforms and integrating them on to a single chip. In addition to the fundamental transducer or sensing element, it is also necessary to integrate sample delivery, excitation sources and detectors [9-12].

One very robust approach is based on an embedded waveguide sensor integrated in a microfluidic chamber. To enable specificity in the sensing platform, a sandwich fluorescent immunoassay is typically used. In this approach, the surface of the waveguide is functionalized with one antibody which is specific to the molecule of interest (antigen) and which is used to pull the antigen out of solution. Subsequently, a secondary fluorescently labeled antibody is exposed to the waveguide and binds to the antigen. The evanescent field of the waveguide excites the fluorophore and this emission provides the detection signal. By using the sandwich pair on the antigen, the specificity of the detection is improved [13-15].

Conventionally, this emitted fluorescent signal is detected by monitoring the light radiated above the waveguide. However, as researchers are pursuing more integrated schemes, this approach has proved problematic. New, fully integrated methods to collect and detect fluorescence signals could lead to pathways that enable these sensing platforms to become more compact lab on a chip (LOC) devices.

One phenomenon that could provide a pathway for the necessary simplification of these systems is based on the backcoupling of the fluorescent signal into the waveguide. The dipole radiation from a bound fluorescent molecule typically radiates each photon in a single, non-controlled direction. When placed above a dielectric stack, dipoles such as a bound fluorescent molecule can radiate preferentially in one direction or another. Depending on the refractive index contrast between the waveguide and the fluorophore, this radiated light can preferentially couple into the waveguide as well, resulting in a detectable signal [16-17]. This back-coupling could allow higher efficiency data collection and enable more fully integrated devices. However, current waveguiding devices 
which exhibit this effect are based on stacked dielectric waveguides. Therefore, subtle changes in the refractive index contrast, caused by environmental changes, can significantly impact not only the device performance but also the backcoupling effect. As such, the ideal detection method will depend greatly on the refractive index contrast. Additionally, a rigorous theoretical model which describes this effect and the regimes of operation for waveguides has not been developed.

Fig. 1 schematically demonstrates the two paths of fluorescent radiation which can be monitored. In the present work, fluorescent measurements are taken using both the conventional method (monitoring fluorescence from above, vertical emission) and the back-coupling method (power transmitted through the waveguide, end emission) as shown in Fig. 1. A complementary theoretical model based on 2D and 3D FDTD simulations is also developed. The results are compared to the theoretical predictions and used to determine the optimum detection route for different index regimes.

Additionally, a variation on the classic fluorescent measurement is performed. Instead of measuring the total fluorescence emitted vertically, the emission is quantified along the waveguide in a spatiotemporal manner. Specifically, the bright area of fluorescent radiation that slowly propagates down the length of the waveguide as the fluorescent dye bleaches is monitored. Using these measurements, fluorescent decay curves are created. This measurement approach provides additional information as compared to the more conventional cumulative technique and could allow for selective excitation of different regions of the waveguide.

\section{EXPERIMENTAL METHODS AND MODELING}

\section{A. Computer Modeling and Simulations}

We modeled the waveguide devices using both $2 \mathrm{D}$ and $3 \mathrm{D}$ Lumerical finite difference time domain (FDTD) software. FDTD simulations solve for a numerical solution to Maxwell's equations as light propagates in time. By using a simulation method that takes into account time-variation, we were able to run simulations to investigate the mechanism of the fluorophore coupling into the waveguide. In addition, the amount of light which was coupled was quantified.

The device geometry was defined using the experimentally provided design specifications (Fig. 2). In addition, to account for variations in fabrication, we varied the number of dipoles, dipole spacing, dipole orientation, cladding thickness, and cladding index and sensing well index according to Table I. To minimize computation time, the majority of the simulations were performed in 2D. However, to ensure the accuracy of the $2 \mathrm{D}$ simulations, a threedimensional simulation was also performed using the exact parameters of the devices provided, and similar results were obtained.

For our simulations, we used dipole radiation sources operating at $642 \mathrm{~nm}$ to model the emitting fluorophores. The device was monitored over a length (L) of $500 \mu \mathrm{m}$ directly around the sensing well region (Fig. 2). The length scale of
$500 \mu \mathrm{m}$ was chosen because we wanted to allow the light coupled into the waveguides to settle into a steady-state propagating mode. We monitored the total power the fluorophores emitted, as well as the power radiated above the waveguide, below the waveguide, and radiated to the left and right within the waveguide. The placement of the power monitors in the simulation can be seen in Fig. 2. It is important to note that the power monitors extend $0.2 \mu \mathrm{m}$ into the cladding in order to capture power from the evanescent field of the guided mode, which also extends approximately $0.2 \mu \mathrm{m}$ beyond the waveguide core. The waveguide core $\left(\mathrm{n}_{2}\right)$ was modeled as silicon nitride with a refractive index of 1.98 , and the cladding $\left(\mathrm{n}_{1}\right)$ was modeled as silica with a refractive index of 1.4355 .

In the initial simulations, we varied the distance between adjacent dipoles (D) and the relative angle of the dipoles $\left(\phi_{1}\right.$ and $\phi_{2}$ ). This additional component to the modeling allowed us to study how the interactions between individual fluorophores can affect the overall coupling efficiency into the waveguide. We ran simulations with 10 dipoles oriented in three different patterns. For these simulations, the thickness of the cladding layer (t) was varied from 8 to $12 \mathrm{~nm}$, and the refractive index of the cladding above the waveguide $\left(\mathrm{n}_{1}\right)$ was varied from 1.4 to 1.6. These thickness and refractive index ranges were selected as they represent the tolerance on the material deposition. Finally, we ran simulations with 10 dipoles in a fixed orientation, in which we varied the refractive index of the sensing well $\left(\mathrm{n}_{3}\right)$. We chose the refractive index ranges as they cover the typical range in biological solutions.

All 2D simulations were run with a minimum mesh size of $0.25 \mathrm{~nm}$ and a simulation length of $3 \mathrm{ps}$. Because we ran 2D simulations, the accuracy of the simulations was set to 3 in order to have accurate results but allow the simulations to finish within a reasonable amount of time. The simulations were run using Lumerical's sweep function. For these sweeps, we only monitored the transmitted power through each of the power monitors, including the transmission box monitor surrounding the dipoles. The transmission box monitor was included to measure the total power being emitted by the fluorophores, which was used for normalizing our results.

\section{B. Device Design and Fabrication}

The embedded waveguide devices were fabricated using the procedure described in previous work [18], with one additional step. In order to accurately measure the emission of the fluorophores from the end using the schematic shown in Fig. 3, it was necessary to cleave the end of the devices using a dicing saw followed by polishing, thus exposing both the input and output ends of the waveguide. The waveguides are approximately $8.6 \mathrm{~mm}$ long after the cleaving step. The main body of the waveguide is $100 \mu \mathrm{m}$ wide and $0.2 \mu \mathrm{m}$ thick. As shown in the schematic in Fig. 2, the devices have a sensing well or region of the device where the upper cladding has been mostly removed, enabling the evanescent excitation of fluorophores. The sensing well is approximately $4.6 \mathrm{~mm}$ long. 


\section{Experimental Setup and Measurements}

To simulate the emission from a fluorescently labeled biolayer, we used LD-700 perchlorate laser dye dissolved in toluene. The laser dye absorbs at $658 \mathrm{~nm}$ and emits at around $680 \mathrm{~nm}$ when dissolved in toluene. This dye was selected due to its robustness and high quantum yield in both air and liquid environments [19]. Two different sets of experiments were performed to verify the simulation results. In the first set, the dye was suspended in solution, and in the second set, the dye was deposited on the surface. This approach allowed two distinctly different refractive index contrasts to be studied. Additionally, in the case of the dry film experiments, the dye is located in the precise region where one would expect fluorescence-tagged molecules to bind to a functionalized device used for biosensing. We determined that the evanescent field of the fundamental mode of the waveguide extends approximately $0.2 \mu \mathrm{m}$ beyond the waveguide core, which is more than enough penetration into the sensing well to excite the fluorescent dye.

To compare the emission which is coupled into the waveguide and the emission which is radiated from the top, a complementary pair of measurements is performed. In the first, the power which is transmitted through the waveguide is measured and in the second the power which is radiated from the top of the device is measured. For all measurements, light from a $658 \mathrm{~nm}$ laser (Thorlabs, $14 \mathrm{~mW} \max$ power) was coupled into the waveguide device using a lensed fiber $(\mathrm{OZ}$ Optics) (Fig. 3). Top- and side-view cameras are used to monitor and align the lensed fiber.

To isolate the dye emission from the coupled laser light, a bandpass filter (ThorLabs, $680 \mathrm{~nm}$ center wavelength, $10 \mathrm{~nm}$ FWHM) was placed between the end facet of the waveguide and a fiber-coupled spectrograph (Andor), which was used to monitor the transmitted power from end emission (Fig. 3a). To measure the power radiated perpendicular to the waveguide (vertical emission), the spectrograph tip was placed directly over the waveguide, with the notch filter located between the waveguide and the spectrograph fiber (Fig. 3b). In both experimental approaches, data was acquired at a rate of 1 sample/second using a background scan to normalize the data and remove any pump laser light from the data that was not filtered out by the bandpass filter. Using the dry films, additional imaging experiments were performed to study the spatiotemporal excitation of the dye. These measurements utilized the top-view camera with the notch filter to monitor the fluorescence. The camera settings were optimized (1 frame per second, $997.56 \mathrm{~ms}$ exposure time, and $5 \mathrm{MHz}$ pixel clock) in order to effectively capture the fluorescent signal on video. We analyzed this data using a custom LabView program that measured the intensity of columns of pixels at every horizontal pixel position on the camera image for every frame of a given video. The result is that we can measure the fluorescence decay of horizontal positions on the waveguide with a resolution of one pixel. This means that the resolution of this analysis is limited only by the resolution of the camera used to obtain the video. Furthermore, we can also visualize how the bright fluorescence area travels down the waveguide length and measure its propagation rate.

In addition to the fluorescence measurements, we also characterized the transmission loss of every device before use to check for fabrication errors or imperfections. For this measurement, we focused light from the output of the waveguide onto a power meter (Thorlabs) using an aspheric lens. We compared the output power from the waveguide to the output power from the lensed fiber to determine transmission loss plus coupling loss.

\section{RESULTS AND DISCUSSION}

The transmission loss measurements verified the uniformity of the device fabrication process across wafers. Utilizing a simulation to estimate the coupling loss from the lensed fiber into the waveguide, we calculated the transmission loss of the waveguides to be approximately $0.3 \mathrm{~dB} / \mathrm{cm}$.

The primary results from the modeling are summarized in Fig. 4-6. The data is shown as the percentage of optical power coupled into the waveguide (propagating to either the left or the right of the dipoles, end emission), the percentage of optical power radiated above the waveguide (vertical emission) and the percentage of optical power radiated below the waveguide, into the substrate. The percentage is calculated by dividing the transmitted power from the monitors around the waveguide and above the waveguide by the total power radiating from the dipoles in the simulation. Calculating these percentages allows us to directly compare different points in the same simulation sweep, for which the dipole orientations are different. It also gives us a unitless number which we can directly compare to the experimental results.

In Fig. 4, the results from our two-dipole simulation (Table I, Model 1) are shown. From the figure, it is easy to conclude that with just two dipoles, the percentage of power coupled into the waveguides can vary dramatically. Generally speaking, when both dipoles are oriented perpendicularly to the waveguide $\left(\phi=90^{\circ}\right)$, more power is coupled into the waveguide; however, this is not the case for every dipole spacing. This is to be expected, because a dipole radiates much less energy parallel to its axis of oscillation. When the dipole oscillates parallel to the waveguide very little light is radiated in the direction of the propagating modes of the waveguide.

Other than indicating that the distance and angle between two dipoles can have a large impact on the amount of light coupled into the waveguide, these simulations alone do not provide enough insight into the practical operation of the waveguide biosensor device. One unique aspect of the modeling was studying the dependence of the coupling on the placement and orientation of the dipoles. The percentage of power coming out both ends of the waveguide (end emission), the percentage of power radiating above the waveguide (vertical emission), and the percentage of power lost into the substrate below the waveguide is strongly dependent on the relative angle and distance between the dipoles. This indicates that in order to accurately model these devices, we need to consider how they react to a being covered by very many 
fluorophores, not just one or two.

We report the results of our first ten-dipole simulation (Table I, Model 2) in Fig. 5. The orientation pattern of the dipoles is indicated on the graphs with arrows. From this figure, we can see that when multiple dipoles are used, the percentage of power radiating above the waveguide (vertical emission), the percentage of power coupled into the waveguide (end emission), and the percentage of power lost into the substrate below the waveguide are more consistent across variations. In fact, certain variations, such as the thickness of the cladding layer in the sensing well have little to no impact on the distribution of power from the dipoles. Varying the index of refraction of the cladding layer has slightly more impact on the distribution of radiated power, but the variations are still usually less than one percentage point.

These results provide significant insight into both the operation of the waveguide device and the device tolerance to variation in fabrication. For example, small variations in the waveguide film thickness do not affect the overall device performance significantly. The only exception is in the case where all the dipoles are oscillating parallel to the waveguide, in which case the coupling was very small. However, this is an unrealistic scenario, and therefore we can expect that with a large number of randomly oriented fluorophores, these variations will tend to go away, and we will see that an average distribution of radiated dipole light coupled into the waveguide and radiated above the waveguide will emerge similar to the percentages seen in Fig. 5b/e/h.

Additionally, changes in the refractive index of the environment can have an impact. As seen in Fig. 6, when we varied the refractive index of the liquid in the sensing well in a second ten-dipole simulation (Table I, Model 3), the light radiated both above the waveguide and coupled into the waveguide (vertical emission and end emission) both increased. The light radiated below the waveguide decreased. Although the ratio of light radiating above to the light coupled into the waveguide remained fairly constant, the overall efficiency of operation increases since less light is lost into the substrate.

We compared the results of the simulations to the measurements taken with the spectrograph. Since the spectrograph measures light in arbitrary counts, we normalized our data according to the following formula:

$$
O=W /(W+A)
$$

Where the output of the device, $\mathrm{O}$, is equal to the amount of light coming out of the waveguide, W (in counts or percentage), divided by the sum of the amount of light coming out of the waveguide and the amount of light radiated above the waveguide, A (in counts or percentage). Since the data obtained from the simulations is in percentage of total power radiated from the dipoles, using the formula above we can directly compare the output of the device from experimental results to the output from the simulations.

Fig. 7 shows the spectrograph spectra taken from the output of the waveguide (end emission) and from above the waveguide (vertical emission) from the experiments in which the laser dye was suspended in liquid, as well as spectra taken from these locations before the dye solution was applied to the device. You can see from the initial spectra that the pump laser is completely filtered out from the wavelength range where the dye emission peak is, indicating that the signal picked up by the spectrograph is completely from the fluorescent dye. There are some slight artifacts around the pump laser center wavelength (Fig. 7 inset) which arise due to how the spectrograph applies background scans when normalizing data. On the through, with dye and above, with dye lines, dips around the pump center wavelength arise because the dye is absorbing the pump laser, causing the power at those wavelengths to be less than the initial background spectra. From the figure, you can see that dye emission collected from both above the waveguide and the output of the waveguide have a spectral peak at around 680 $\mathrm{nm}$. This is consistent with spectrofluorometer data we took of the dye dissolved in toluene, and means that the spectrograph is measuring only the laser dye emission.

From this data, we can obtain the amount of light that is radiated above the waveguide (vertical emission) and the amount that couples into the waveguide (end emission). Because the bandpass filter cuts off a portion of the fluorescence peak, we did not integrate the peak to obtain the amount of light above the waveguide and through the waveguide. Instead, we used the number of counts at the fluorescence peak as an indicator of the amount of light radiated in each direction. The peak counts for light radiated above the waveguide is about 8100 , and the peak counts for light coupled into the waveguide is about 1400 . In order to directly compare the simulation and experiment, we double the counts collected from the waveguide, as we only collected light travelling in one direction in the waveguide, not in both directions as in the simulation. From these peak counts, we can calculate the output, $\mathrm{O}$, as defined in (1), to be 0.257 , and the output from the simulations ranges from 0.278 to 0.327 .

As you can see from the results, there is good experimental agreement with the simulations. This confirms our simulation model and indicates just how much light one can expect to couple into the waveguide mode for this device. These results also give insight on ways to increase the efficiency of the performance of this device. For example, the alignment and spacing of the fluorophores relative to the waveguide is very significant. By designing a surface functionalization that would create an optimal spacing between the fluorophores and from the cladding surface to the flurophores, one could expect to see performance gains.

One reason there is a slight disparity between the simulation results and the experimental results is due to a difference of the refractive index of the liquid in the sensing well in the simulation and the experiment. Additionally, differences arise due to a limited numerical aperture of the spectrograph tip. Due to the spectrograph's limited numerical aperture, alignment was very important when conducting experiments and slight misalignments caused some experimental error. Alignment error and the refractive index 
difference of the liquid in the sensing well easily account for the difference in output between the simulations and experiments.

The data in Fig. 7 is only from measurements taken while the laser dye was still suspended in toluene. We found that when we let the toluene dry into a film, the back-coupling signal was below the detection threshold of the spectrograph. Therefore, the only measurements we made with dry films of laser dye were the spatiotemporal fluorescence measurements.

Fig. 8 shows spatiotemporal fluorescence measurements taken with a camera and analyzed using a custom LabView program we developed. These measurements were performed with a dry film of laser dye. In Fig. 8a, time vs. normalized intensity is shown at different positions along the waveguide. In each graph, you can see a fluorescence excitation and decay curve, and you can see that the position of this curve is shifted back in time for positions further down the waveguide. This indicates that the fluorescent dye absorbs nearly all of the light at a given position, and it is not until that fluorescent emission begins to decay (the dye begins to bleach) that the pump 658 $\mathrm{nm}$ laser light can travel further down the waveguide. Additionally, it appears that there are two distinct decay regimes that appear at all the different positions on the waveguide. While the reason for these two regimes is unknown, it is possible that they are simply a characteristic of the dye itself. When fit to an exponential decay curve, the two regions tend to have slightly different time constants. This could be indicative of different paths of bleaching within the dye [20-21].

In Fig. 8b, position vs. normalized intensity graphs are shown at various points of time during the experiment. In each graph, you can see where the bulk of the emitted fluorescence is on the waveguide. Looking at all the graphs, it is clear that the peak fluorescence moves down the waveguide as time progresses. This further indicates that the fluorescent laser dye is absorbing nearly all the pump $658 \mathrm{~nm}$ light until it begins to bleach, allowing dye further down the waveguide to absorb the light. It is interesting to note that the shape of the fluorescence peak remains relatively unchanged as it propagates down the length of the waveguide. Also, after the main peak passes, there are a few brighter spots left on the waveguide, which are likely due to clumps of dye that formed when the liquid dried on the surface of the device. These bright spots and some other noise indicate that the dye probably did not completely bleach over the course of this experiment. Given the clear dependence on the fluorescence properties of dye in Fig. 8, it is straightforward to conclude that the density of the dye on the surface of the device also plays a role.

These spatiotemporal measurements demonstrate the usefulness of our system for a number of interesting fluorescent-dye based applications [22-24]. For example, the device and measurement system could be used to characterize decay rates of fluorescent dyes operating in various application-specific scenarios. One could conjugate a dye with biomolecules, surface-functionalize the device for use as a sensor, and measure how the fluorescent dye bleaches under specific sets of conditions. This type of measurement could provide valuable insight into how signals from waveguidebased fluorescence biosensors change over time. Additionally, one could use these spatiotemporal fluorescence measurements to perform cascading assays, in which the waveguide sensor is functionalized for different analytes along its length. As the fluorescence spot propagates down the waveguide, you could expect to see different signals for each analyte present in solution. This type of measurement opens up the possibility for very complex multiplexing on a single sample or device that could be useful in detection of complex analytes or signatures in a given solution sample. Complex multiplexing and rapid fluorescence characterization are attractive for simultaneously improving utility and cost of LOC devices.

\section{CONCLUSIONS}

We have developed and verified a rigorous theoretical model for both the back-coupling and the vertical radiating detection schemes for embedded waveguide sensors. Using two extremes of refractive index contrast, we demonstrated that the stacked dielectric waveguides could be operated in either mode; however, depending on the type of experiment, one approach may have advantages over the other. Furthermore, we have demonstrated that the back-coupling effect is very constant with respect to fabrication tolerances. This makes leveraging this effect attractive for many different kinds of sensing applications, including further miniaturization of sensing platforms, which is desirable for truly LOC devices.

Finally, we have developed a system to perform spatiotemporal measurements using the unique geometry of the waveguide biosensor device in conjunction with a fluorescent laser dye. Utilizing these measurements, we are able to produce fluorescence decay curves at arbitrary points along the length of the waveguide, which could be useful for determining the fluorescent characteristics of fluorescent dyes or labels in various environments. We envision that these spatiotemporal fluorescence measurements could also be used to achieve more complex multiplexing and cascading assays. These complex assays could greatly expand the scope of optofluidic and biophotonic sensing devices and LOC devices in general [24-25].

\section{REFERENCES}

[1] A. M. Armani, R. P. Kulkarni, S. E. Fraser, R. C. Flagan, and K. J. Vahala, "Label-free, single-molecule detection with optical microcavities," Science, vol. 317, pp. 783-787, Aug 2007.

[2] F. S. Ligler, "Perspective on Optical Biosensors and Integrated Sensor Systems," Analytical Chemistry, vol. 81, pp. 519-526, Jan 2009.

[3] V. M. N. Passaro, C. de Tullio, B. Troia, M. La Notte, G. Giannoccaro, and F. De Leonardis, "Recent Advances in Integrated Photonic Sensors," Sensors, vol. 12, pp. 15558-15598, Nov 2012.

[4] A. L. Washburn and R. C. Bailey, "Photonics-on-a-chip: recent advances in integrated waveguides as enabling detection elements for real-world, lab-on-a-chip biosensing applications," Analyst, vol. 136, pp. 227-236, 2011.

[5] D. Erickson, S. Mandal, A. H. J. Yang, and B. Cordovez, "Nanobiosensors: optofluidic, electrical and mechanical approaches to biomolecular detection at the nanoscale," Microfluidics and Nanofluidics, vol. 4, pp. 33-52, Jan 2008. 
[6] J. Wang, "Electrochemical biosensors: Towards point-of-care cancer diagnostics," Biosensors \& Bioelectronics, vol. 21, pp. 1887-1892, Apr 2006.

[7] A. H. Liu, T. Watanabe, I. Honma, J. Wang, and H. S. Zhou, "Effect of solution $\mathrm{pH}$ and ionic strength on the stability of poly(acrylic acid)encapsulated multiwalled carbon nanotubes aqueous dispersion and its application for NADH sensor," Biosensors \& Bioelectronics, vol. 22, pp. 694-699, Dec 2006.

[8] N. Lloret, R. S. Frederiksen, T. C. Moller, N. I. Rieben, S. Upadhyay, L. De Vico, J. H. Jensen, J. Nygard, and K. L. Martinez, "Effects of buffer composition and dilution on nanowire field-effect biosensors," Nanotechnology, vol. 24, Jan 2013.

[9] A. Crespi, Y. Gu, B. Ngamsom, H. Hoekstra, C. Dongre, M. Pollnau, R. Ramponi, H. H. van den Vlekkert, P. Watts, G. Cerullo, and R. Osellame, "Three-dimensional Mach-Zehnder interferometer in a microfluidic chip for spatially-resolved label-free detection," Lab on a Chip, vol. 10, pp. 1167-1173, 2010.

[10] C. Dongre, R. Dekker, H. Hoekstra, M. Pollnau, R. Martinez-Vazcluez, R. Osellame, G. Cerullo, R. Ramponi, R. van Weeghel, G. A. J. Besselink, and H. H. van den Vlekkert, "Fluorescence monitoring of microchip capillary electrophoresis separation with monolithically integrated waveguides," Optics Letters, vol. 33, pp. 2503-2505, Nov 2008.

[11] D. Duval, A. B. Gonzalez-Guerrero, S. Dante, J. Osmond, R. Monge, L. J. Fernandez, K. E. Zinoviev, C. Dominguez, and L. M. Lechuga, "Nanophotonic lab-on-a-chip platforms including novel bimodal interferometers, microfluidics and grating couplers," Lab on a Chip, vol. 12, pp. 1987-1994, 2012.

[12] M. Medina-Sanchez, S. Miserere, and A. Merkoci, "Nanomaterials and lab-on-a-chip technologies," Lab on a Chip, vol. 12, pp. 1932-1943, 2012.

[13] G. L. Duveneck, A. P. Abel, M. A. Bopp, G. M. Kresbach, and M. Ehrat, "Planar waveguides for ultra-high sensitivity of the analysis of nucleic acids," Analytica Chimica Acta, vol. 469, pp. 49-61, Sep 2002.

[14] H. Mukundan, A. S. Anderson, W. K. Grace, K. M. Grace, N. Hartman, J. S. Martinez, and B. I. Swanson, "Waveguide-Based Biosensors for Pathogen Detection," Sensors, vol. 9, pp. 5783-5809, Jul 2009.

[15] C. A. Rowe, S. B. Scruggs, M. J. Feldstein, J. P. Golden, and F. S. Ligler, "An array immunosensor for simultaneous detection of clinical analytes," Analytical Chemistry, vol. 71, pp. 433-439, Jan 1999.

[16] H. Hoekstra and H. B. H. Elrofai, "Theory of optical spontaneous emission rates in layered structures," Physical Review E, vol. 71, Apr 2005.

[17] L. Polerecki, J. Hamrle, and B. D. MacCraith, "Theory of the radiation of dipoles placed within a multilayer system," Applied Optics, vol. 39, pp. 3968-3977, Aug 2000.

[18] R. Duer, R. Lund, R. Tanaka, D. A. Christensen, and J. N. Herron, "InPlane Parallel Scanning: A Microarray Technology for Point-of-Care Testing," Analytical Chemistry, vol. 82, pp. 8856-8865, Nov 2010.

[19] T. F. Johnston, R. H. Brady, and W. Proffitt, "Powerful single-frequency ring dye-laser spanning the visible spectrum," Applied Optics, vol. 21, pp. 2307-2316, 1982.

[20] C. Eggeling, J. Widengren, L. Brand, J. Schaffer, S. Felekyan, and C. A. M. Seidel, "Analysis of photobleaching in single-molecule multicolor excitation and forster resonance energy transfer measurement," Journal of Physical Chemistry A, vol. 110, pp. 2979-2995, Mar 2006.

[21] T. Gensch, M. Bohmer, and P. F. Aramendia, "Single molecule blinking and photobleaching separated by wide-field fluorescence microscopy," Journal of Physical Chemistry A, vol. 109, pp. 6652-6658, Aug 2005.

[22] M. Lehnhardt, T. Riedl, T. Rabe, and W. Kowalsky, "Room temperature lifetime of triplet excitons in fluorescent host/guest systems," Organic Electronics, vol. 12, pp. 486-491, Mar 2011.

[23] M. J. Lochhead, K. Todorof, M. Delaney, J. T. Ives, C. Greef, K. Moll, K. Rowley, K. Vogel, C. Myatt, X. Q. Zhang, C. Logan, C. Benson, S. Reed, and R. T. Schooley, "Rapid Multiplexed Immunoassay for Simultaneous Serodiagnosis of HIV-1 and Coinfections," Journal of Clinical Microbiology, vol. 49, pp. 3584-3590, Oct 2011.

[24] S. Mandal, J. M. Goddard, and D. Erickson, "A multiplexed optofluidic biomolecular sensor for low mass detection," Lab on a Chip, vol. 9, pp. 2924-2932, 2009.

[25] H. K. Hunt and A. M. Armani, "Label-Free Biological and Chemical Sensors," Nanoscale, vol. 2, pp. 1544-1559, 2010.

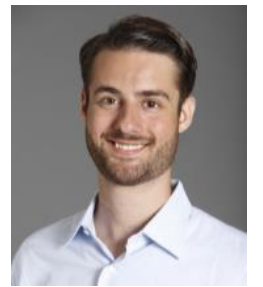

Mark C. Harrison received his BS and MS degrees in electrical engineering from the University of Southern California, Los Angeles in 2010 and 2012, respectively.

Since 2010, he has been a PhD student in the Ming Hsieh Department of Electrical Engineering - Electrophysics in the Viterbi School of Engineering at the University of Southern California, working in the Armani Research Group. His research interests include developing novel uses for integrated optical devices in the areas of biodetection and communications.

Mr. Harrison's awards and honors include the National Defense Science and Engineering Graduate Fellowship and the University of Southern California's Provost's Fellowship. $\mathrm{He}$ is a student member of SPIE and IEEE.

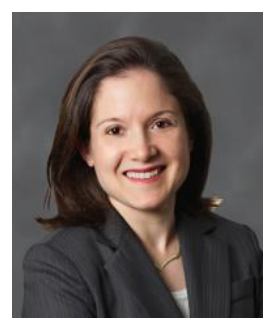

Andrea M. Armani received her BA degree in physics from the University of Chicago in 2001 and her PhD in Applied Physics with a minor in Biology from the California Institute of Technology in 2007. From 2006 to 2008, she was the Clare Boothe Luce Post-doctoral Fellow in Chemical Engineering and Biology at the California Institute of Technology.

Since 2008, she has been the Fluor Early Career Chair of Engineering and an Assistant Professor in the Mork Family Department of Chemical Engineering and Materials Science in the Viterbi School of Engineering at the University of Southern California. She has courtesy appointments in both Electrical Engineering and Biomedical Engineering, enabling her research group to explore device development and characterization as well biodetection experiments. She is a coauthor of more than 60 peer-reviewed journal articles and conference proceedings. She is also an Associate Editor of Optics Letters.

Prof. Armani's awards and honors include the Office of Naval Research Young Investigator Award, the Presidential Early Career Award for Excellence in Science and Engineering, and the NIH New Innovator Award. She is a senior member of SPIE and IEEE. 
TABLE I

VALUES OF KEY SIMULATION PARAMETERS

\begin{tabular}{cccccccc}
\hline \hline $\begin{array}{c}\text { Mod } \\
\mathrm{el}\end{array}$ & $\mathrm{t}(\mu \mathrm{m})$ & $\begin{array}{c}\mathrm{D} \\
(\mu \mathrm{m})\end{array}$ & $\begin{array}{c}\# \\
\text { dipoles }\end{array}$ & $\phi_{1}\left({ }^{\circ}\right)$ & $\phi_{2}\left({ }^{\circ}\right)$ & $\mathrm{n}_{1}$ & $\mathrm{n}_{3}$ \\
\hline 1 & 0.010 & $0.1-0.8$ & 2 & $0-90$ & 90 & 1.4355 & 1.33 \\
2 & $0.008-$ & 0.1 & 10 & $90,90,0$ & 90,0, & $1.4-1.6$ & 1.33 \\
& 0.012 & & & & 0 & & \\
3 & 0.010 & 0.1 & 10 & 90 & 0 & 1.4355 & $1-1.4$ \\
\hline \hline
\end{tabular}

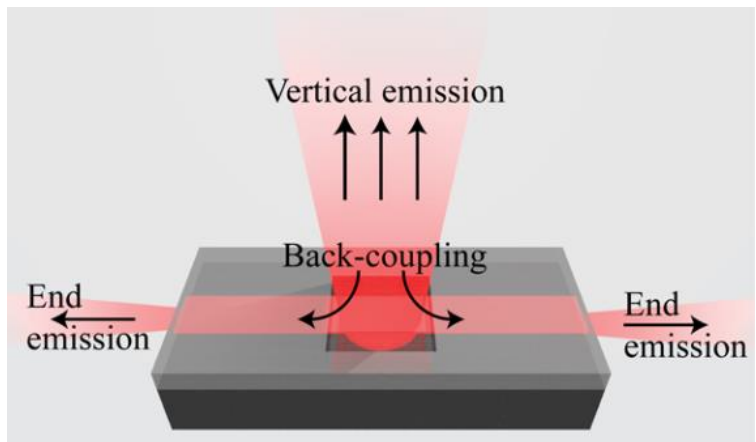

Fig. 1. Schematic diagram indicating emission paths for fluorescent radiation which can be experimentally monitored. Fluorophores in the sensing well can radiate vertically above the device or their radiation can back-couple into the waveguide resulting in end emission. This diagram does not show the laser light used to excite the fluorophores.

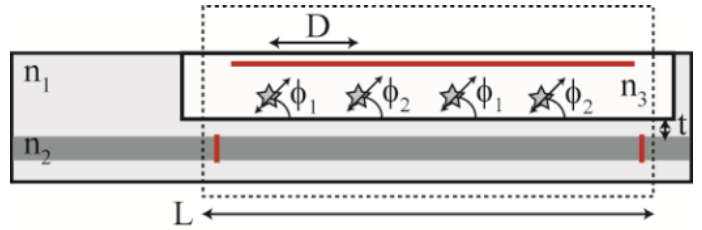

Fig. 2. Schematic diagram of device geometry used for simulations. D is distance between dipoles, $\phi_{1}$ and $\phi_{2}$ are angles of alternating dipoles, $t$ is the thickness of the cladding in the sensing well, $\mathrm{L}$ is the length of the simulation, and $\mathrm{n}_{1}, \mathrm{n}_{2}$, and $\mathrm{n}_{3}$ are the refractive indices of the cladding, waveguide core, and sensing well, respectively. The locations of power monitors in the simulation are indicated by red lines, and the extent of the simulation region is indicated by a dashed line.

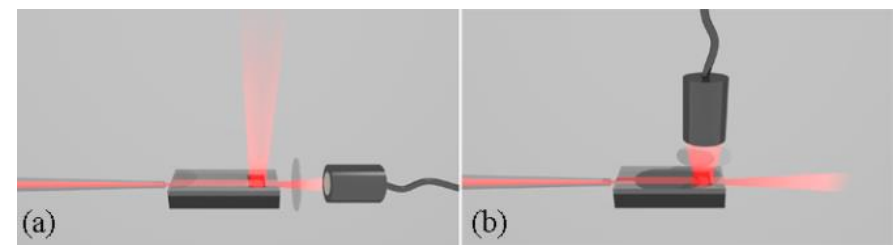

Fig. 3. Schematic of testing set-up. Light is coupled into the device from the left via a lensed fiber, and output is collected with a filter and spectrograph a) from the output of the waveguide and $b$ ) from above the sensing well of the device.

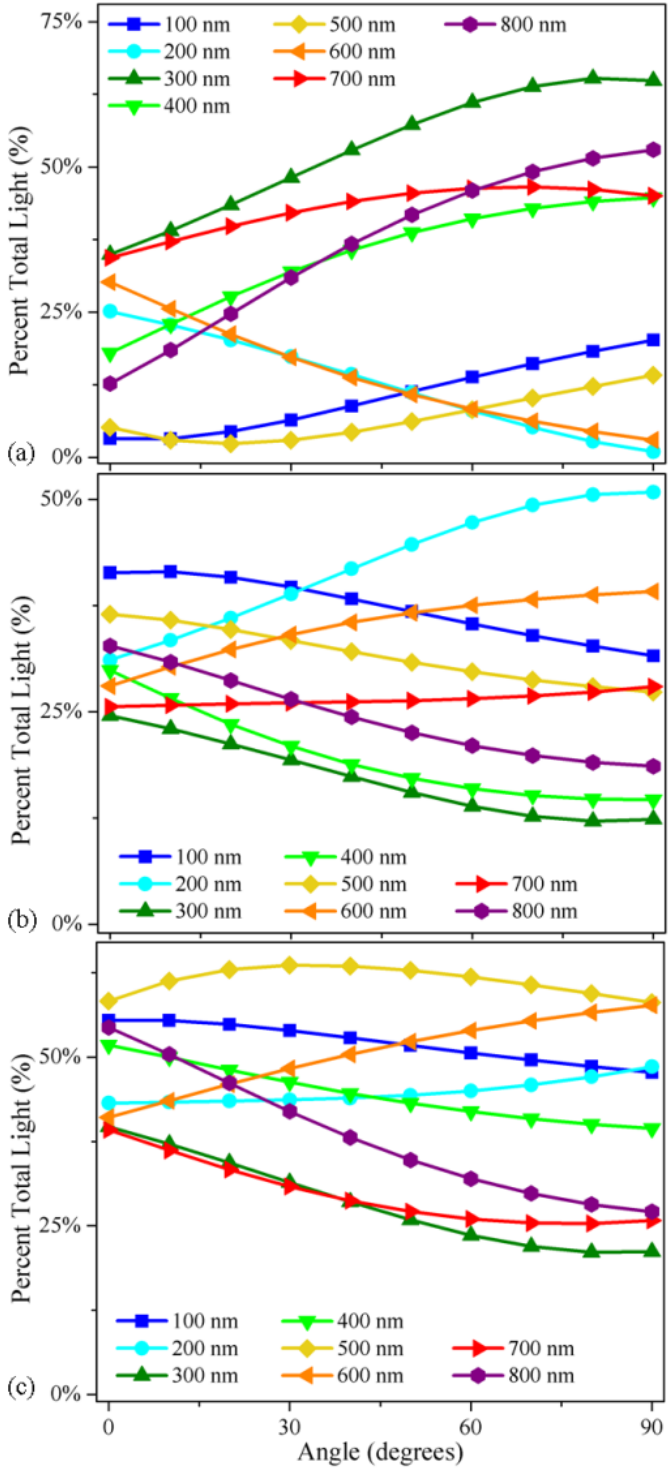

Fig. 4. Results from 2-dipole simulation (Model 1). Graphs show, for different dipole spacing and angles, the percentage of optical power a) coupled into the waveguide, b) radiating above the waveguide, and c) lost into the substrate below the waveguide.
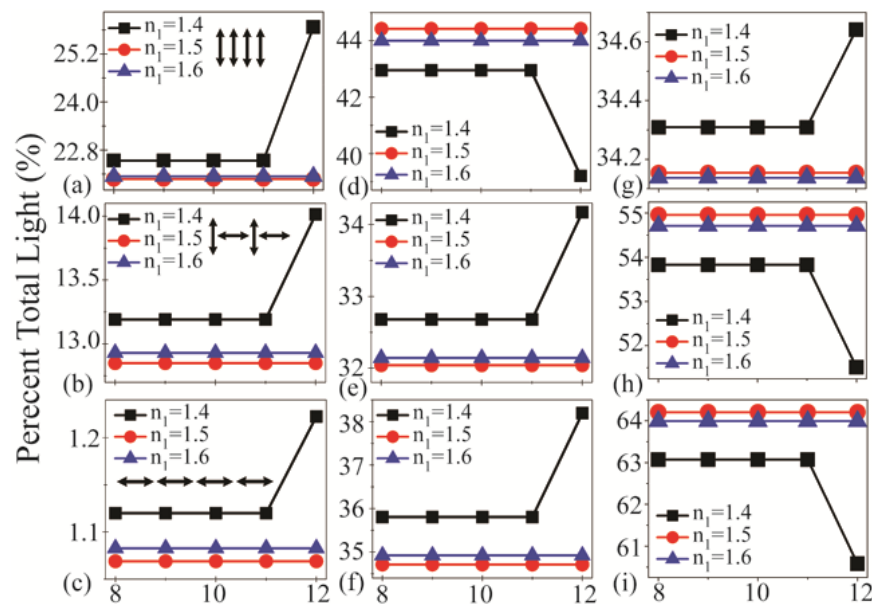

Cladding Thickness (nm)

Fig. 5. Results from the first 10-dipole simulation (Model 2). Graphs show the percentage of optical power a), b), c) back-coupled into the waveguide, d), 
e), f)radiating above the waveguide and g), h), i) lost into the substrate below the waveguide for different dipole orientations. The dipole orientation patterns are indicated by arrows on the graphs in parts a-c.

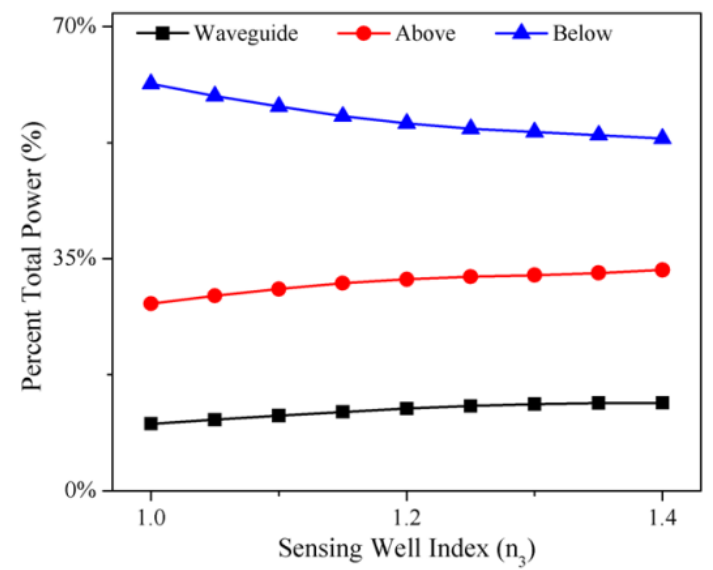

Fig. 6. Results from the second 10-dipole simulation (Model 3). Percentage of total power coupled into the waveguide, radiated above the waveguide, and lost into the substrate below the waveguide for different refractive indices of the sensing well.

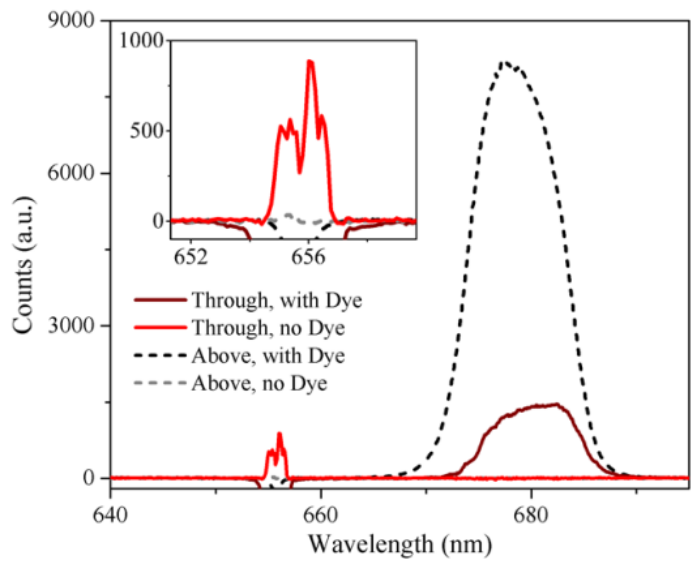

Fig. 7. Spectrograph spectra taken from the output of the waveguide (through), and above the waveguide (above), with and without fluorescent dye on the device. Inset shows a close-up of the wavelength region around the center wavelength of the pump laser.
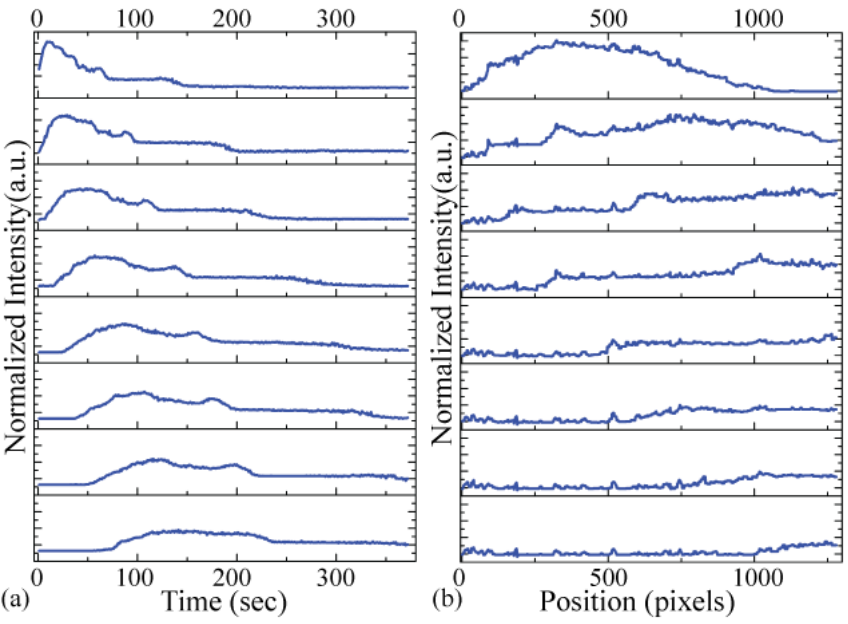

Fig. 8. Spatiotemporal measurements of the fluorescent dye film on the device. The bright fluorescent area slowly propagated down the length of the waveguide, and a) shows normalized intensity as a function of time for various positions on the waveguide, while b) shows normalized intensity vs. position on the waveguide for various times during the experiment. In a) the graphs are in order of position down the waveguide, whereas in b) the graphs are in order of increasing time during the experiment. 